\title{
Computation of Autonomous Safety Maneuvers Using Segmentation and Optimization
}

\section{Pavel Anistratov}


Linköping Studies in Science and Technology Licentiate Thesis No. 1860

\title{
Computation of Autonomous Safety Maneuvers Using Segmentation and Optimization
}

\author{
Pavel Anistratov
}


Linköping Studies in Science and Technology

Licentiate Thesis No. 1860

This is a Swedish Licentiate Thesis.

Swedish postgraduate education leads to a Doctoral degree and/or a Licentiate degree.

A Doctoral degree comprises 240 ECTS credits (4 years of full-time studies).

A Licentiate degree comprises 120 ECTS credits,

of which at least 60 ECTS credits constitute a Licentiate thesis.

Pavel Anistratov

pavel.anistratov@liu.se

www. vehicular.isy.liu.se

Division of Vehicular Systems

Department of Electrical Engineering

Linköping University

SE-581 33 Linköping, Sweden

Copyright (C) 2019 Pavel Anistratov, unless otherwise noted.

All rights reserved.

Anistratov, Pavel

Computation of Autonomous Safety Maneuvers

Using Segmentation and Optimization

ISBN 978-91-7929-947-7

ISSN 0280-7971

Typeset with $\mathrm{ITT}_{\mathrm{E}} \mathrm{X} 2_{\varepsilon}$

Printed by LiU-Tryck, Linköping, Sweden 2019 


\section{ABSTRACT}

This thesis studies motion planning for future autonomous vehicles with main focus on passenger cars. By having automatic steering and braking together with information about the environment, such as other participants in the traffic or obstacles, it would be possible to perform autonomous maneuvers while taking limitations of the vehicle and road-tire interaction into account. Motion planning is performed to find such maneuvers that bring the vehicle from the current state to a desired future state, here by formulating the motion-planning problem as an optimal control problem. There are a number of challenges for such an approach to motion planning; some of them are how to formulate the criterion in the motion planning (objective function in the corresponding optimal control problem), and how to make the solution of motion-planning problems efficient to be useful in online applications. These challenges are addressed in this thesis.

As a criterion for motion-planning problems of passenger vehicles on doublelane roads, it is investigated to use a lane-deviation penalty function to capture the observation that it is dangerous to drive in the opposing lane, but safe to drive in the original lane after the obstacle. The penalty function is augmented with certain additional terms to address also the recovery behavior of the vehicle. The resulting formulation is shown to provide efficient and steady maneuvers and gives a lower time in the opposing lane compared to other objective functions. Under varying parameters of the scenario formulation, the resulting maneuvers are changing in a way that exhibits structured characteristics.

As an approach to improve efficiency of computations for the motion-planning problem, it is investigated to segment motion planning of the full maneuver into several smaller maneuvers. A way to extract segments is considered from a vehicle dynamics point of view, and it is based on extrema of the vehicle orientation and the yaw rate. The segmentation points determined using this approach are observed to allow efficient splitting of the optimal control problem for the full maneuver into subproblems.

Having a method to segment maneuvers, this thesis further studies methods to allow parallel computation of these maneuvers. One investigated method is based on Lagrange relaxation and duality decomposition. Smaller subproblems are formulated, which are governed by solving a low-complexity coordination problem. Lagrangian relaxation is performed on a subset of the dynamic constraints at the segmentation points, while the remaining variables are predicted. The prediction is possible because of the observed structured characteristics resulting from the used lane-deviation penalty function. An alternative approach is based on adoption of the alternating augmented Lagrangian method. Augmentation of the Lagrangian allows to apply relaxation for all dynamic constraints at the segmentation points, and the alternating approach makes it possible to decompose the full problem into subproblems and coordinating their solutions by analytically solving an overall coordination problem. The presented decomposition methods allow computation of maneuvers with high correspondence and lower computational times compared to the results obtained for solving the full maneuver in one step. 



\section{ACKNOWLEDGMENTS}

First I would like to thank my main supervisor Lars Nielsen for letting me start this journey of post-graduate studies. Björn Olofsson and Jan Åslund have helped me as my co-supervisors. Lars and Björn are acknowledged for being my co-authors and guiding me through research challenges. Björn's extensive help with proofreading and guidance in scientific writing is very much appreciated.

I would like to thank the people at the Division of Vehicular Systems for providing a nice working environment. Thank you, the frequent visitors of the fika room, for interesting discussions during coffee breaks and for contributing to my listening comprehension of the Swedish language. Friday fika pastries are as well appreciated.

Thanks to Xavier Llamas, Sergii Voronov, Victor Fors, Fatemeh Mohseni, Mahdi Morsali, Vaheed Nezhadali, and others who helped me with tips about life in Sweden. Xavi is appreciated for being around during summers when there are almost no people at the division. Sergii is appreciated for being my language companion. I would like to thank all the people who helped me with my Swedish courses. Thanks to Mattias Tiger and Björn for letting me practice my language communication skills with them, and others who continued talking in Swedish even after my replies in English.

Thanks to Victor for showing me around when I was new at the division, for being a nice colleague to share office with, and for being a reliable and knowledgeable partner in the course assignments and the project course work. It was pleasant to travel together for conferences, study trips, and to have a vacation together. Thank you for introducing me to the world of desktop Linux.

Erik Frisk, Christofer Sundström, Daniel Jung, Jan, and Björn are acknowledged for keeping me busy with teaching activities and helping me to develop my teaching skills. Thanks to Kristoffer Ekberg and Sergii for helping me to start with teaching sessions and labs. Kristoffer's organized activities are appreciated. His initiative to participate in Blodomloppet as a team motivated me to start training for running and the afterwork events were also nice to attend.

This work was partially supported by the Wallenberg AI, Autonomous Systems and Software Program (WASP) funded by the Knut and Alice Wallenberg Foundation. The students from the first batch of the WASP Autonomous Systems graduate school are acknowledged for the pleasant time during our joint courses and study trips. Organization efforts for these activities from the WASP seniors are also appreciated.

Thanks to my old friends for not forgetting me. I had fun whenever we traveled together, you visited me, or I visited you in return. I would also like to acknowledge my parents, my brother, and my other relatives for supporting me on my way. Thank you very much for that!

Linköping, November 2019

Pavel Anistratov 



\section{Contents}

1 Introduction 1

1.1 Background . . . . . . . . . . . . . . . . 2

1.1.1 Optimization for Safety-Critical Motion Planning . . . . . 3

1.1.2 Computational Aspects of Optimization . . . . . . . . . 3

1.2 Thesis Focus . . . . . . . . . . . . . . . . . . . 4

1.3 Contributions . . . . . . . . . . . . . . . . . . 5

1.4 Future Research . . . . . . . . . . . . . . . . . . 8

$\begin{array}{lr}\text { References } & 9\end{array}$

$\begin{array}{ll}\text { Papers } & 13\end{array}$

I Extended Lane-Deviation Penalty for Autonomous Vehicle Avoidance Maneuvers with Recovery Behavior 15

1 Introduction . . . . . . . . . . . . . . . . 16

2 Modeling . . . . . . . . . . . . . . . . . 17

2.1 Chassis Model . . . . . . . . . . . . . . . . . . . . . 17

2.2 Wheel Dynamics . . . . . . . . . . . . . . . . . . . . . 17

2.3 Tire Model . . . . . . . . . . . . . . . . . . . . . . . . . . 19

2.4 Modeling of Road and Obstacle . . . . . . . . . . . . 19

3 Baseline Formulation of Optimal Control Problem . . . . . . . . 22

3.1 Formulation . . . . . . . . . . . . . . . . 22

3.2 Implementation . . . . . . . . . . . . . . . . . 24

3.3 Initialization of Numerical Optimization . . . . . . . . . 24

3.4 Maneuvers for the Baseline LDP for Different Initial Velocities 24

4 Extension of the Optimization Formulation for Recovery Behavior 25

4.1 Velocity Recovery . . . . . . . . . . . . . . . . 25

4.2 Driving in the Middle of the Lane . . . . . . . . . . 26

4.3 Force Distribution . . . . . . . . . . . . . . . . . 28

4.4 Behavior for Higher Initial Velocities . . . . . . . . . . 28

4.5 Complete Penalty Function for Improved Recovery Behavior 29

5 Analysis of Singularities from Actuators to Motion in the Model 30

5.1 Theoretical Analysis Using the Jacobian . . . . . . . . . 30 
5.2 Analysis for Complete Tire Model . . . . . . . . . . . . . . 32

6 Scenario Variations . . . . . . . . . . . . . . . . . . 34

6.1 Obstacle Width ................... . . . 34

6.2 Obstacle Length . . . . . . . . . . . . . . . . . . . . . . . . . . . 35

6.3 Initial Velocity . . . . . . . . . . . . . . . . 35

6.4 Distance to the Obstacle . . . . . . . . . . . . . . 35

7 LDP Comparisons and Variations . . . . . . . . . . . . . 38

7.1 Minimum-Time Formulation . . . . . . . . . . . . . . . . 38

7.2 Quadratic Deviation Penalty Formulation . . . . . . . . . 39

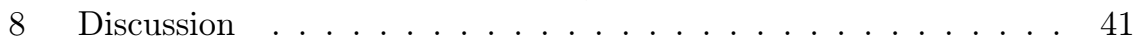

8.1 Generality of Recovery Behavior . . . . . . . . . . . . . 41

8.2 Singularity Analysis Using the Jacobian . . . . . . . . . . . 42

8.3 Structure in the Solutions . . . . . . . . . . . . . . . 42

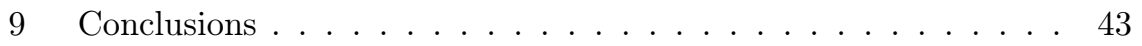

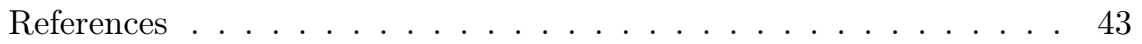

II Segmentation and Merging of Autonomous At-the-Limit Ma$\begin{array}{ll}\text { neuvers for Ground Vehicles } & 47\end{array}$

1 Introduction . . . . . . . . . . . . . . . . . . . . . 48

2 Modeling and Optimization . . . . . . . . . . . . . . . . 48

2.1 LDP for Double Lane-Change Maneuver . . . . . . . . . . 49

2.2 LDP for Turn Maneuver . . . . . . . . . . . . . . . . . . . 49

3 Splitting Method: Description .............. . 49

3.1 Splitting Points . . . . . . . . . . . . 50

3.2 Start and Final Constraints . . . . . . . . . . . . . . 51

3.3 What are Good Splitting Points? . . . . . . . . . . . 52

4 Results ..................... . . 52

4.1 Trajectory Reconstruction . . . . . . . . . . . . . 52

4.2 Trajectory Computation by Interpolation . . . . . . . . . 56

4.3 Constraint Relaxation . . . . . . . . . . . . . . 59

5 Discussion ..................... 60

6 Conclusions ............................ 61

References ....................... 62

III Efficient Motion Planning for Autonomous Vehicle Maneuvers Using Duality-Based Decomposition 65

1 Introduction . . . . . . . . . . . . . . . . . 66

2 Problem Formulation as a Separable MinMax Problem . . . . . . 67

2.1 Primal Problem . . . . . . . . . . . . . . . . 67

2.2 Dual Problem . . . . . . . . . . . . . . . . 68

2.3 Dual-Function Gradient . . . . . . . . . . . . . . . . . . . 69

2.4 Computational Approach . . . . . . . . . . . . 70

3 Model and Optimization Problem . . . . . . . . . . . . 71

3.1 Vehicle Model . . . . . . . . . . . . . . . . . 71

3.2 Tire Model . . . . . . . . . . . . . . . . 71 
3.3 Optimization Problem . . . . . . . . . . . . . . . . . . . 72

4 Algorithm . . . . . . . . . . . . . . . . . . . 72

4.1 Overview of the Algorithm . . . . . . . . . . . . 73

4.2 Implementation Details . . . . . . . . . . . . . . 75

5 Results ...................... . . 75

5.1 Parameters of the Model . . . . . . . . . . . . . . 75

5.2 Formulation of Segmented Maneuvers in CasADi . . . . . . 76

5.3 Comparison of Full and Segmented Maneuvers . . . . . . . 76

5.4 Comparison with the Segmentation-Merging Method . . . 76

5.5 Performance Results . . . . . . . . . . . . . . . . . 78

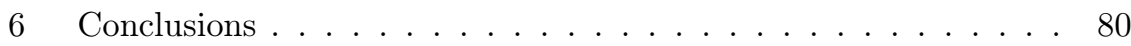

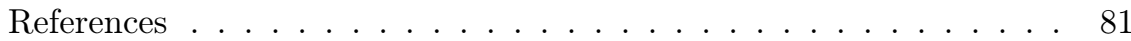

IV Autonomous-Vehicle Maneuver Planning Using Segmentation and the Alternating Augmented Lagrangian Method 83

1 Introduction . . . . . . . . . . . . . . . . . 84

2 Vehicle Model . . . . . . . . . . . . . . . . . . . . . 85

2.1 Time Domain . . . . . . . . . . . . . . . . . . . 85

2.2 Road Coordinates . . . . . . . . . . . . . . . . . . 85

2.3 Tire Model . . . . . . . . . . . . . . . . . . . . . . . . . . 87

2.4 Lane-Deviation Penalty Function . . . . . . . . . . . . 87

3 Separable Optimal Control Problem . . . . . . . . . . . . . . 88

3.1 Continuous Formulation . . . . . . . . . . . . . . . . . 88

3.2 Discretization of Cost Function and Vehicle Dynamics . . . 88

3.3 Separable Discretized Formulation . . . . . . . . . . . . . 89

3.4 Applying Alternating Augmented Lagrangian Method . . . 90

4 Parameters and Implementation . . . . . . . . . . . . . . . . . 91

4.1 Road Definition and Parameters . . . . . . . . . . . . . . 91

4.2 Model and Problem Parameters . . . . . . . . . . . . . . 92

4.3 Comments on Implementation . . . . . . . . . . . . . . . 92

4.4 Initialization . . . . . . . . . . . . . . . . . . . . 92

4.5 Scaling . . . . . . . . . . . . . . . . . . . 93

4.6 Solver Configuration . . . . . . . . . . . . . . . . . . . 93

$5 \quad$ Results . . . . . . . . . . . . . . . . . . . . 93

5.1 Solution of the Decomposed Problem . . . . . . . . . . 94

5.2 Time Performance . . . . . . . . . . . . . . . . . . . . 96

5.3 Importance of Scaling . . . . . . . . . . . . . . . . . . . 97

6 Conclusion . . . . . . . . . . . . . . . . . . . . . . . 99

References ........................ . . 99 


\section{Chapter 1}

\section{Introduction}

The dream of autonomous operation of ground vehicles has been around for some time, see, e.g., the video from GM's Motorama exhibit in 1956 (King Rose Archives, 2013), where a family is depicted to travel on a highway in an autonomous car in the then future 1976. As of today, autonomous passenger cars are not yet fully available. Some people are still cautious about the idea of driverless cars because not all aspects defining coexistence of people and autonomous vehicles are yet resolved. However, it seems that interest in the area is taking a new breath, with people talking about it during their coffee breaks, established automobile scientific conferences moving focus, and research and education programs being funded.

Different reasons and goals are put forward as driving forces for the development. Driving safety is often mentioned as there is a desire to have no fatal accidents on public roads (see, e.g., VisionZero (Trafikverket, 2019) for Sweden). Another area is underground mining, where one waits for harmful gases to clear after blasting to minimize risk of negative effects for humans. With autonomous vehicles, it is expected that vehicles could be run almost immediately after the explosion without risk for people. Further, higher coverage, extended possibilities, and decreased costs of public transportation are yet additional motives behind research on autonomous vehicles.

The improvements in the area are possible because of new technical solutions, improved sensors, and more powerful computational resources available onboard. Autonomous vehicles are benefiting from machine learning, new approaches to software development, and other areas. Modern cars are more like computers with new approaches to product development, where remote updates and continuous development approaches are possible, even though high software dependence brings concerns regarding testing and safety.

The topic of autonomous vehicles is huge and this thesis is aiming to target one specific area of it, namely the area of motion planning for ground vehicles in 


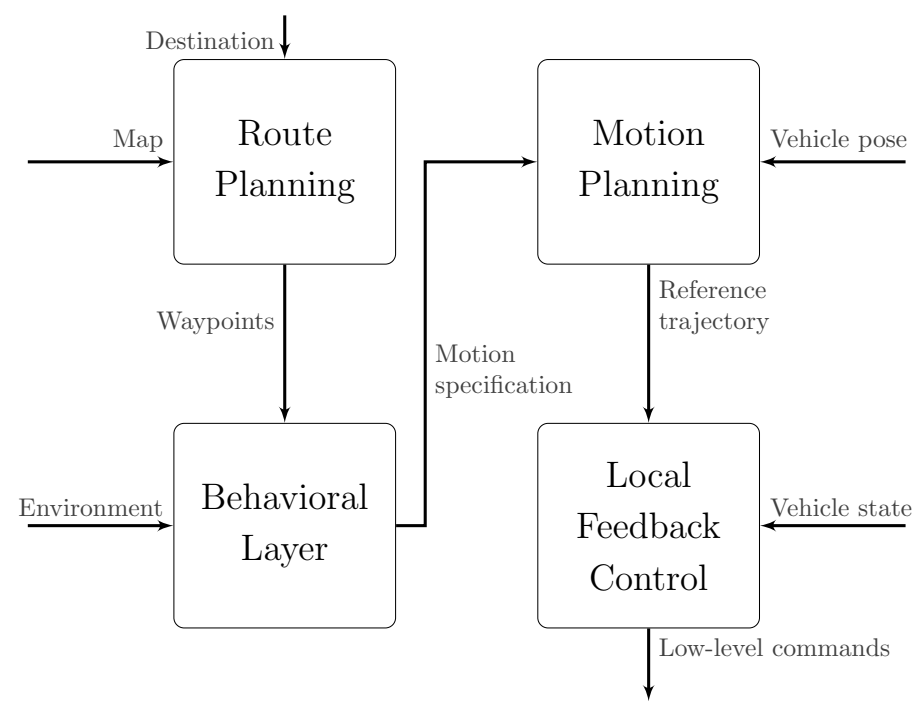

Figure 1.1: Hierarchy of a decision-making process used in driverless cars (adopted from (Paden et al., 2016)).

safety-critical situations. To put motion planning in a context, one could refer to the hierarchy of a decision-making process for autonomous cars presented in (Paden et al., 2016), see Figure 1.1. According to that hierarchy, the responsibility of a motion planner is to provide a reference path or trajectory given motion specifications determined by higher levels. Multiple approaches are existing to do motion planning. A brief overview of the area is given in the following section.

\subsection{BACKGROUND}

A recent survey of motion planning and control techniques in autonomous vehicles is found in the previously mentioned (Paden et al., 2016), where the hierarchy of a decision-making process used in driverless cars is presented as Route Planning, Behavioral Decision Making, Motion Planning, and Local Feedback Control. Paden et al. (2016) define the motion-planning layer to be responsible for computing a safe, comfortable, and dynamically feasible trajectory from the current configuration of the vehicle to the goal configuration provided by the Behavioral Decision Making level. Several approaches are outlined for path/trajectory planning, including approaches based on graph-search methods (e.g., motion primitives used to plan on a state lattice (Likhachev and Ferguson, 2009, Ljungqvist, 2019)), the RRT method and its extensions (LaValle, 2006, Karaman and Frazzoli, 2011), and variational (optimization) methods (Limebeer and Rao, 2015, Bergman, 2019). Kelly (2017) gives an introduction to interior- 
point methods for trajectory optimization. These methods have found broad application nowadays. However, they were in limited use for a long time, see, e.g., (Forsgren et al., 2002) for a brief history of the methods.

Motion planning is also possible to approach by considering an artificial potential field to guide the vehicle (Gerdes and Rossetter, 2001). This approach is experimentally validated in (Rossetter et al., 2004) for lane-keeping driver assistance. Ji et al. (2017) have recently extended the idea by including an MPC applied after the path has been computed using the defined artificial potential field. By considering simplified models and assuming limited coupling between the longitudinal and lateral dynamics (Nilsson et al., 2015) in the motion planning, Nilsson et al. (2016) obtain lane-change maneuvers on highways with low computational resources using a quadratic programming formulation of the optimization problem.

\subsubsection{Optimization for Safety-Critical Motion Planning}

Results of offline optimization problems are fruitful in understanding and designing safety systems, see, e.g., the survey on optimal control in automotive applications (Sharp and Peng, 2011). Examples on the use of offline optimization methods include: a study of at-the-limit maneuvers and a comparison of vehicle models of different complexity used in optimizations (Berntorp et al., 2014); an analysis of minimum distance when an avoidance maneuver is still possible (Shiller and Sundar, 1998); or the utilization of an optimal-control based method for quantifying the maneuverability of autonomous vehicles during emergency highway-speed situations (Dingle and Guzzella, 2010). Fors et al. (2018) have formulated and interpreted optimal braking patterns using optimization, to provide new insights for potential future safety systems with adaptation of the level of braking. Using the concept of attainable force volumes of optimal maneuvers, Fors et al. (2019) discuss a set of control principles for lane-keeping control with close to optimal behavior.

Optimal control results for a simplified vehicle model (particle model) have been used as reference value by the control system to, e.g., perform an avoidance maneuver by applying the modified Hamiltonian algorithm (Gao et al., 2019) for nonlinear optimal control allocation or to prevent road departure on curved roads (Gao and Gordon, 2019). An evasive maneuver assist for collision mitigation with oncoming vehicles has been developed in (Arikere et al., 2018) based on optimal control results for a particle model, and a subsequent controller design is presented.

\subsubsection{Computational Aspects of Optimization}

The use of optimization-based methods is often connected with computational challenges. For offline applications, these challenges are not as significant compared to online applications, and there are many useful applications only requiring offline computation. A number of different approaches improving online 
applicability of optimization-based methods have been proposed in the literature.

The load of the motion-planning problem could be divided between the offline and online parts. Instead of defining motion constraints directly as optimization constraints, it is possible to define the motion-planning problem as a search for a suitable combination of motion primitives, which are pre-computed offline (Frazzoli et al., 2005, Pivtoraiko and Kelly, 2011). Several approaches have been studied to use a pre-computed offline trajectory in an online model predictive controller: to calculate the required steering angle for vehicles on slippery roads with the highest possible entry speed when the trajectory is known (Falcone et al., 2007); to perform local trajectory re-planning for autonomous off-road ground vehicles (Yoon et al., 2009); or to control a racing-car with a high re-planning frequency and a long planning horizon (Subosits and Gerdes, 2019).

A motion-planning problem could be decoupled into path planning and subsequent trajectory planning (LaValle, 2006), i.e., by first finding a path and then a suitable velocity profile. Kapania et al. (2016) consider a racing application where a sequential two-step algorithm alternating between path and velocity profile optimization is used to generate minimum-time trajectories. $\mathrm{Xu}$ et al. (2012) apply graph search based on predefined maneuvers and then optimize the resulting path by using polynomial approximations of the trajectories.

The original motion-planning problem could be decomposed into several connected subproblems, which potentially are easier to solve. Paper III investigates one of these approaches using the concept of duality decomposition presented in (Lasdon, 1968). Another decomposition method is the alternating direction method of multipliers, which is covered in (Boyd et al., 2011). The mentioned approaches are targeting the situation when the objective function of the resulting optimization is decomposable.

Other approaches to improve online applicability of optimization-based methods are based on making the computational process faster. This could be achieved by improving the computational performance of the underlying methods, e.g., obtaining a solution of the linear system of equations arising at each step of many iterative optimization solvers faster (Cao et al., 2016), or by not fully solving the optimization problem at each step when a sequence of slightly changing problems are computed. For interior-point methods, this could be achieved by early termination based on a small number of maximum iterations, or not updating the barrier penalty parameter, which also allows taking advantage of warm starting the solver (Wang and Boyd, 2010).

\subsection{Thesis Focus}

By considering each maneuver as a sequence of shorter sub-maneuvers bringing the vehicle from one state to another, the goal of this thesis is to study the motion planning with two foci. The first one is on obtaining the aforementioned submaneuvers as maneuver segments. The second one is on formulating the motionplanning problem as a sequence of maneuver segments to potentially benefit in 
computation time and in better understanding of the complete maneuver nature. The particular characteristics of the maneuver segments are of course dependent on the objective in the motion-planning optimization formulation. Development of a problem formulation based on the idea of an objective function with smooth transitions from one sub-maneuver to the next is also given consideration. The focus of that formulation is on decreasing the time in the opposing lane in double lane-change maneuvers.

\subsection{COntributions}

The publications by the author are the following:

- Pavel Anistratov, Björn Olofsson, and Lars Nielsen. Lane-Deviation Penalty for Autonomous Avoidance Maneuvers. In: 14th International Symposium on Advanced Vehicle Control. Beijing, China, 2018.

- Pavel Anistratov, Björn Olofsson, and Lars Nielsen. Segmentation and Merging of Autonomous At-the-Limit Maneuvers for Ground Vehicles. In: 14th International Symposium on Advanced Vehicle Control. Beijing, China, 2018.

- Pavel Anistratov, Björn Olofsson, and Lars Nielsen. Efficient Motion Planning for Autonomous Vehicle Maneuvers Using Duality-Based Decomposition. In: 9th IFAC Symposium on Advances in Automotive Control. Orleáns, France, 2019.

The first publication has been substantially extended, and the extended manuscript is included in this thesis as Paper I. The other two publications are included as Papers II and III, respectively. Paper IV presents research submitted as a paper to a conference.

The additional manuscripts by the author are thus the following:

- Pavel Anistratov, Björn Olofsson, and Lars Nielsen. Extended LaneDeviation Penalty for Autonomous Vehicle Avoidance Maneuvers with Recovery Behavior. Manuscript.

- Pavel Anistratov, Björn Olofsson, Oleg Burdakov, and Lars Nielsen. Autonomous Vehicle Maneuver Planning Using Segmentation and the Alternating Augmented Lagrangian Method. Submitted to conference.

The main contributions of the included papers are summarized in the following.

Paper I: Extended Lane-Deviation Penalty for Autonomous Vehicle Avoidance Maneuvers with RECOVERY BEHAVIOR

The paper considers a double lane-change maneuver as a safety-critical maneuver requiring at-the-limit operation. The observation that it is dangerous for the 


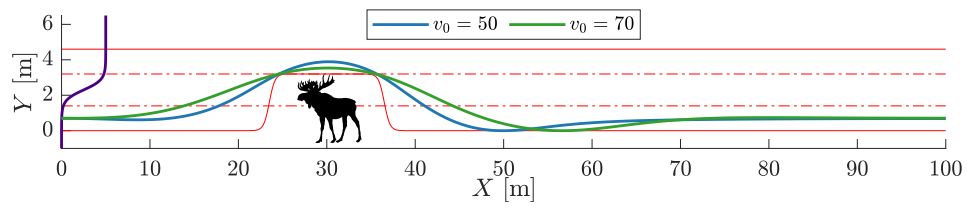

Figure 1.2: Examples from Paper I of double lane-change maneuvers using the presented formulation for two different initial velocities $(\mathrm{km} / \mathrm{h})$.
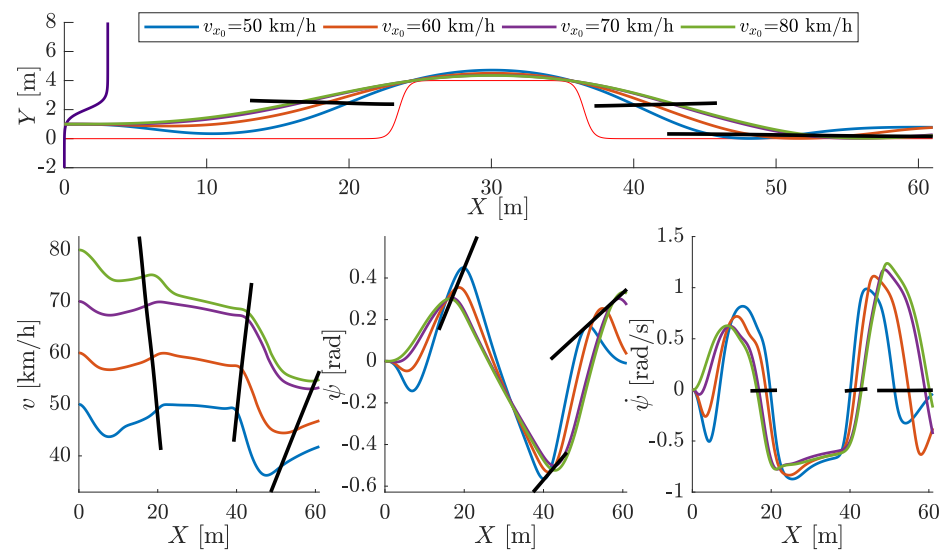

Figure 1.3: Illustration of the splitting concept from Paper II.

vehicle to be in the opposing lane, but it is safe to drive in the original lane again after the obstacle, is captured using a lane-deviation penalty (LDP) objective function. Additional penalty terms are included in the final objective function to improve the recovery behavior after the obstacle, motivated by an analysis of the relation between actuation and vehicle motion. For varying parameters of the problem scenario, such as the initial speed of the vehicle (see Figure 1.2 for examples) and parameters of the obstacle, the formulation is observed to result in efficient and steady maneuvers, while keeping the time in the opposing lane at a minimum.

\section{Paper II: Segmentation and Merging of Autonomous At-The-Limit Maneuvers for Ground Vehicles}

The paper considers how to split a full maneuver into maneuver segments and investigates how to decrease the complexity of motion-planning optimizations using a segmentation approach. The segmentation points are used to set constraints at segment junctions for several smaller optimization problems comprising parts of the full maneuver. The technique is illustrated for a double lane-change maneuver (see Figure 1.3 for an illustration of the splitting concept) and for 


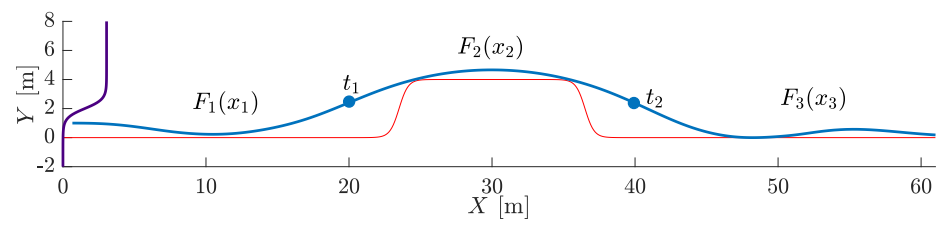

Figure 1.4: Illustration of a maneuver splitted into three segments/subproblems from Paper III.

a $90^{\circ}$-turn maneuver. It is shown that when a priori information about the maneuver is available in terms of a small subset of values of the state variables at the splitting points, a close to optimal maneuver is possible to obtain by applying the presented segmentation and merging technique.

\section{Paper III: Efficient Motion Planning for Autonomous Vehicle Maneuvers Using Duality-Based Decomposition}

The approach to segmented maneuvers from Paper II is adapted and extended in this paper to allow parallel computation of maneuver segments for improved computational performance. The original optimization problem is modified, such that certain variables at the splitting points are precomputed and thus fixed, while the remaining variables are obtained by performing Lagrange relaxation of the coupling constraints. Figure 1.4 shows an example of a maneuver splitted into three segments. The presented method is evaluated in a safety-critical double lane-change situation and is for certain initialization strategies shown to improve computational performance.

\section{Paper IV: Autonomous Vehicle Maneuver Planning Using Segmentation and the Alternating Augmented LAGRANGIAN METHOD}

Compared to Paper III, the original optimization problem is modified by adopting the alternating augmented Lagrangian method. The approach to segmented maneuver computation in this paper is made to be no longer dependent on precomputed data at the splitting points. This is achieved by solving a lowcomplexity initialization problem and by moving all coupling constraints to the coordination problem, which allows parallel computation of the segmented maneuvers. The coordination problem is possible to solve quickly using an analytical approach. For the evaluated example of a double lane-change maneuver, it is shown that an acceptable solution is obtained after just a few alternating iterations (see Figure 1.5), with sequentially improved solutions obtained after more iterations if necessary. 


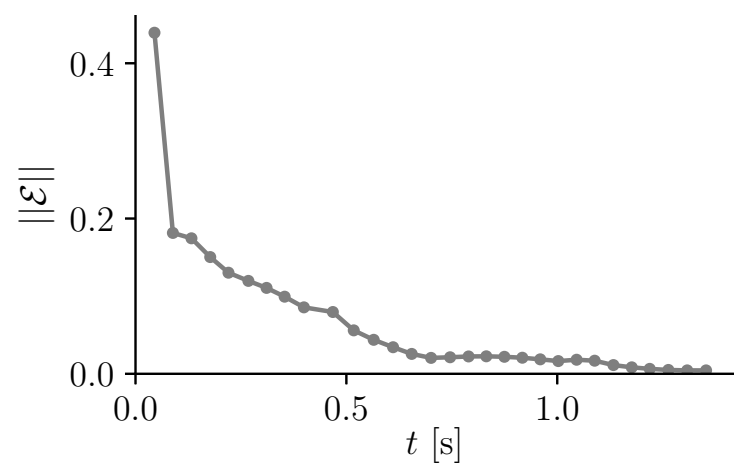

Figure 1.5: Error-norm measure of the coupling constraints $\|\mathcal{E}\|$ over computational time for the evaluated example from Paper IV. Each point is one alternating iteration. Rapid decrease of the error after just a few iterations is obtained.

\subsection{Future Research}

In this thesis, a double lane-change maneuver has been used as an example of a safety-critical maneuver requiring operation at the limit of the tire-road friction. A possible step in future research is to make an attempt to generalize and extend the application of the presented ideas on maneuver segmentation and decomposition techniques to other safety maneuvers. Analysis of computational and convergence aspects, e.g., with respect to number of segments in the decomposition, is relevant for further development of the methods towards practical applications. Moreover, models of different fidelity could be considered and compared when the decomposition techniques are applied.

It is also of interest to target online motion planning where the problem formulation is updated sequentially based on the current position of the vehicle and detected obstacles. To this purpose, the ideas on problem decomposition presented in the last two papers could be extended to an online scenario. In such a scenario, the motion planner adaptively segments the motion-planning problem. When formulating a new motion-planning problem, the planner can reuse maneuver segments computed for previous motion-planning problems, and also re-initialize the solvers (to save initialization time) for the respective subproblem, corresponding to already traversed segments, for improved computational performance. 


\section{References}

Arikere, A., Yang, D., Klomp, M., and Lidberg, M. (2018). Integrated evasive manoeuvre assist for collision mitigation with oncoming vehicles. Vehicle System Dynamics, 56(10), 1577-1603.

Bergman, K. (2019). On Motion Planning Using Numerical Optimal Control. Licentiate Thesis No. 1843, Division of Automatic Control, Faculty of Science \& Engineering, Linköping University.

Berntorp, K., Olofsson, B., Lundahl, K., and Nielsen, L. (2014). Models and methodology for optimal trajectory generation in safety-critical road-vehicle manoeuvres. Vehicle System Dynamics, 52(10), 1304-1332.

Boyd, S., Parikh, N., Chu, E., Peleato, B., and Eckstein, J. (2011). Distributed optimization and statistical learning via the alternating direction method of multipliers. Foundations and Trends in Machine Learning, 3, 1-122.

Cao, Y., Seth, A., and Laird, C.D. (2016). An augmented lagrangian interiorpoint approach for large-scale NLP problems on graphics processing units. Computers $\&$ Chemical Engineering, 85, 76-83.

Dingle, P. and Guzzella, L. (2010). Optimal emergency maneuvers on highways for passenger vehicles with two- and four-wheel active steering. In American Control Conference, 5374-5381. Baltimore, MD, USA.

Falcone, P., Borrelli, F., Asgari, J., Tseng, H.E., and Hrovat, D. (2007). Predictive active steering control for autonomous vehicle systems. IEEE Transactions on Control Systems Technology, 15(3), 566-580.

Fors, V., Olofsson, B., and Nielsen, L. (2018). Formulation and interpretation of optimal braking and steering patterns towards autonomous safety-critical manoeuvres. Vehicle System Dynamics, 57(8), 1206-1223.

Fors, V., Olofsson, B., and Nielsen, L. (2019). Attainable force volumes of optimal autonomous at-the-limit vehicle manoeuvres. Vehicle System Dynamics. doi:10.1080/00423114.2019.1608363. 
Forsgren, A., Gill, P.E., and Wright, M.H. (2002). Interior methods for nonlinear optimization. SIAM Review, 44(4), 525-597.

Frazzoli, E., Dahleh, M.A., and Feron, E. (2005). Maneuver-based motion planning for nonlinear systems with symmetries. IEEE Transactions on Robotics, 21(6), 1077-1091.

Gao, Y. and Gordon, T. (2019). Optimal control of vehicle dynamics for the prevention of road departure on curved roads. IEEE Transactions on Vehicular Technology, 68(10), 9370-9384.

Gao, Y., Gordon, T., and Lidberg, M. (2019). Optimal control of brakes and steering for autonomous collision avoidance using modified Hamiltonian algorithm. Vehicle System Dynamics, 57(8), 1224-1240.

Gerdes, J.C. and Rossetter, E.J. (2001). A unified approach to driver assistance systems based on artificial potential fields. Journal of Dynamic Systems, Measurement, and Control, 123(3), 431-438.

Ji, J., Khajepour, A., Melek, W.W., and Huang, Y. (2017). Path planning and tracking for vehicle collision avoidance based on model predictive control with multiconstraints. IEEE Transactions on Vehicular Technology, 66(2), 952-964.

Kapania, N.R., Subosits, J., and Gerdes, J.C. (2016). A sequential two-step algorithm for fast generation of vehicle racing trajectories. Journal of Dynamic Systems, Measurement, and Control, 138(9), 091005.

Karaman, S. and Frazzoli, E. (2011). Sampling-based algorithms for optimal motion planning. The International Journal of Robotics Research, 30(7), 846-894.

Kelly, M. (2017). An introduction to trajectory optimization: How to do your own direct collocation. SIAM Review, 59(4), 849-904.

King Rose Archives (2013). GM Motorama Exhibit 1956. https://www.youtube. com/watch? $\mathrm{v}=\mathrm{Rx} 6 \mathrm{keHpeYak}$. (Date accessed: 2019-10-22).

Lasdon, L. (1968). Duality and decomposition in mathematical programming. IEEE Transactions on Systems Science and Cybernetics, 4(2), 86-100.

LaValle, S.M. (2006). Planning Algorithms. Cambridge University Press, New York, NY, first edition.

Likhachev, M. and Ferguson, D. (2009). Planning long dynamically feasible maneuvers for autonomous vehicles. The International Journal of Robotics Research, 28(8), 933-945.

Limebeer, D. and Rao, A. (2015). Faster, higher, and greener: Vehicular optimal control. Control Systems Magazine, 35(2), 36-56. 
Ljungqvist, O. (2019). On motion planning and control for truck and trailer systems. Licentiate Thesis No. 1832, Division of Automatic Control, Faculty of Science \& Engineering, Linköping University.

Nilsson, J., Brännström, M., Coelingh, E., and Fredriksson, J. (2015). Longitudinal and lateral control for automated lane change maneuvers. In American Control Conference (ACC), 1399-1404. Chicago, IL, USA.

Nilsson, J., Silvlin, J., Brännström, M., Coelingh, E., and Fredriksson, J. (2016). If, when, and how to perform lane change maneuvers on highways. IEEE Intelligent Transportation Systems Magazine, 8(4), 68-78.

Paden, B., Čáp, M., Yong, S.Z., Yershov, D., and Frazzoli, E. (2016). A survey of motion planning and control techniques for self-driving urban vehicles. IEEE Transactions on Intelligent Vehicles, 1(1), 33-55.

Pivtoraiko, M. and Kelly, A. (2011). Kinodynamic motion planning with state lattice motion primitives. In IEEE/RSJ International Conference on Intelligent Robots and Systems, 2172-2179. San Francisco, CA, USA.

Rossetter, E., Switkes, J., and Gerdes, J. (2004). Experimental validation of the potential field lanekeeping system. International Journal of Automotive Technology, 5(2), 95-108.

Sharp, R.S. and Peng, H. (2011). Vehicle dynamics applications of optimal control theory. Vehicle System Dynamics, 49(7), 1073-1111.

Shiller, Z. and Sundar, S. (1998). Emergency lane-change maneuvers of autonomous vehicles. Journal of Dynamic Systems, Measurement, and Control, $120(1), 37-44$.

Subosits, J. and Gerdes, J.C. (2019). From the racetrack to the road: Realtime trajectory replanning for autonomous driving. IEEE Transactions on Intelligent Vehicles, 4(2), 309-320.

Trafikverket (2019). Vision Zero Academy. https://www.trafikverket.se/en/ startpage/operations/Operations-road/vision-zero-academy. (Date accessed: 2019-10-22).

Wang, Y. and Boyd, S. (2010). Fast model predictive control using online optimization. IEEE Transactions on Control Systems Technology, 18(2), $267-278$.

Xu, W., Wei, J., Dolan, J.M., Zhao, H., and Zha, H. (2012). A real-time motion planner with trajectory optimization for autonomous vehicles. In IEEE International Conference on Robotics and Automation, 2061-2067. Saint Paul, MN, USA. 
Yoon, Y., Shin, J., Kim, H.J., Park, Y., and Sastry, S. (2009). Model-predictive active steering and obstacle avoidance for autonomous ground vehicles. Control Engineering Practice, 17(7), 741-750. 
Papers 


\section{Papers}

The papers associated with this thesis have been removed for copyright reasons. For more details about these see:

http://urn.kb.se/resolve?urn=urn:nbn:se:liu:diva-162164 



\section{FACULTY OF SCIENCE AND ENGINEERING}

Linköping Studies in Science and Technology,

Licentiate Thesis No. 1860, 2019

Department of Electrical Engineering

Linköping University

SE-581 83 Linköping, Sweden

www.liu.se 